\title{
Are there Optical Magnons?
}

\author{
U. KÖBLER* \\ Research Centre Jülich, Institute PGI, 52425 Jülich, Germany
}

\begin{abstract}
Optical magnons should occur in magnets containing two in-equivalent magnetic species only. However, Heisenberg interactions between in-equivalent magnetic atoms can be expected to be weak. This is because free exchange of electrons between chemically different magnetic atoms appears not generally possible. To the best of our knowledge optical magnons have never been identified unambiguously experimentally. Confusion is provided by the fact that two magnon branches commonly occur in antiferromagnets with ferromagnetically ordered crystallographic planes and opposite spin orientations from plane to plane. This applies to $\mathrm{MnO}, \mathrm{EuTe}, \mathrm{CoCl}_{2}, \mathrm{Fe}_{2} \mathrm{O}_{3}, \mathrm{~K}_{2} \mathrm{FeF}_{4}$. Associated with the ferromagnetic planes is a particular low-energy magnon branch. The high-energy magnon branch is the antiferromagnetic branch and not an optical magnon. In $\mathrm{Fe}_{3} \mathrm{O}_{4}$ (magnetite), weak interactions between the $\mathrm{Fe}^{2+}$ moments and the $\mathrm{Fe}^{3+}$ moments are evidenced by the fact that the order parameters of the $\mathrm{FeO}$ and of the $\mathrm{Fe}_{2} \mathrm{O}_{3}$ subsystem have different temperature dependencies. The observed two magnon branches can be attributed to the $\mathrm{Fe}_{2} \mathrm{O}_{3}$ and to the $\mathrm{FeO}$ subsystem, respectively. This applies equally to the two observed magnon branches in mixed crystals such as $\mathrm{Rb}_{2} \mathrm{Mn}_{0.5} \mathrm{Ni}_{0.5} \mathrm{~F}_{4}, \mathrm{KCo}_{0.71} \mathrm{Mn}_{0.29} \mathrm{~F}_{3}$ or $\mathrm{Mn}_{0.3} \mathrm{Co}_{0.7} \mathrm{~F}_{2}$ that can be understood as modified dispersions of the constituent materials.
\end{abstract}

DOI: 10.12693/APhysPolA.133.459

PACS/topics: 75.30.Ds, 75.30.Et

\section{Introduction}

Magnons are not the relevant excitations to control spin dynamics. This reveals, for instance, from the fact that magnon dispersions are independent of spin quantum number but thermal decrease of the magnetic order parameter is different in magnets with integer and halfinteger spin [1]. Moreover, thermal decrease of the magnetic order parameter is universal, that is, independent of spin structure while magnon dispersions depend on spin structure. As a consequence, quite opposite to magnons, the relevant excitation spectrum that is responsible for spin dynamics must depend on spin quantum number but is independent of spin structure.

As was concluded by renormalization group theory [2], spin dynamics is controlled by a boson field. In Ref. [3] it was shown, that the field quanta are essentially magnetic dipole radiation emitted by the precessing spins. The field bosons propagate ballistic, independent of the atomistic background. This is the origin of universality of the boson controlled thermodynamic observables. Since integer and half-integer spins precess differently, the generated field quanta are correspondingly different, and the associated boson fields have different energy densities. Thermal decrease of the order parameter is controlled by the heat capacity of the boson field and therefore is universal but depends on whether the spin is integer or half-integer [3].

Magnons and boson fields are not completely independent excitations but can interact. This modifies magnon dispersions, mainly in the low $q$-range $[1,4]$. It is observed that due to attractive interactions between the

*e-mail: u.koebler@fz-juelich.de dispersion relations of magnons and bosons, magnons can assume the dispersion of the bosons at small $q$-values. This opens the possibility to evaluate the dispersions of the field bosons from the known magnon dispersions [4]. Since the bosons propagate ballistic, their dispersion is a simple power function of wave-vector over a large energy and $q$-range [1]. The exponent $x$ of the $q^{x}$ power function is characteristic for the dimensionality of the boson field, and is independent of spin structure but depends on whether the spin is integer or half-integer $[1,4]$. Appearance of the $q^{x}$ term is always associated with a magnon gap. Magnon dispersions at larger $q$-values better conform to spin wave theory in that they are independent of spin quantum number and depend on spin structure. As a consequence, at small and at large $q$-values, magnons have analytically different wave-vector dependence. The two functions of wave vector have different temperature dependencies $[1,4]$.

\section{Experimental details}

An optical magnon branch should result from interactions between chemically different magnetic atoms. Confusion is provided by the fact that two magnon branches can occur in antiferromagnets with ferromagnetically ordered crystallo-graphic planes and opposite spin orientations from plane to plane [5]. This applies to EuTe [5], $\mathrm{K}_{2} \mathrm{FeF}_{4}$ [6], $\mathrm{MnO}$ [7], $\mathrm{CoCl}_{2}, \mathrm{CoBr}_{2}$ [8] and to $\mathrm{Fe}_{2} \mathrm{O}_{3}$ [9]. Figure 1 shows magnon energies of the low $q$ range of the lamellar antiferromagnet $\mathrm{K}_{2} \mathrm{FeF}_{4}$ [6]. As the large magnon gaps indicate, magnon-boson interaction is strong. As a consequence, magnons adapt over a large $q$-range to the $q^{1.25}$ dispersion of the two-dimensional boson field in magnets with integer spin $(S=2)$ [1]. The $2 \mathrm{D}$ boson field is consistent with spin orientations within quadratic basal plane $[1,6]$. The high-energy magnon 
branch hast to be identified as antiferromagnetic branch while the low energy branch as ferromagnetic branch. This becomes evident from the different field dependencies of the two branches (Fig. 2).

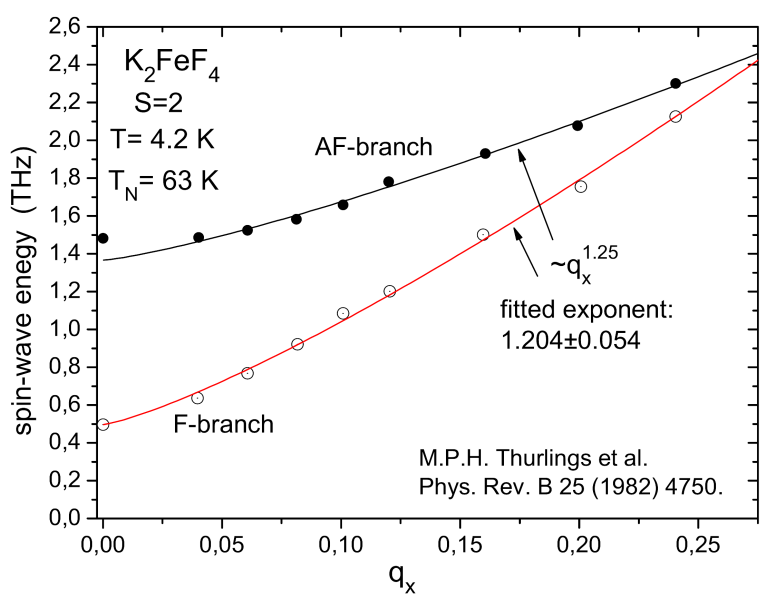

Fig. 1. Magnon dispersions within quadratic basal plane of $\mathrm{K}_{2} \mathrm{FeF}_{4}$ showing low-energy ferromagnetic branch and high-energy antiferromagnetic branch. Both branches have adapted to the $q^{1.25}$ dispersion of the twodimensional boson field in magnets with integer spin $(S=2)[6]$

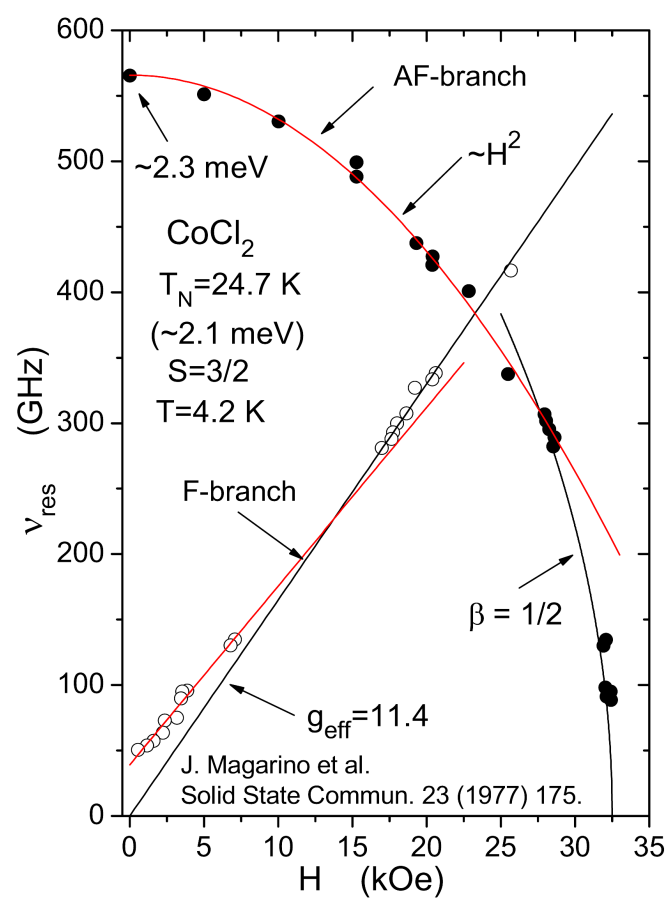

Fig. 2. Field dependence of the gap energies of the antiferromagnetic (filled points) and of the ferromagnetic (circles) magnon branch of $\mathrm{CoCl}_{2}$ at $T=4.2 \mathrm{~K}$ $\left(T_{N}=24.7 \mathrm{~K}\right)$ measured by AFMR [8]. The energy of the antiferromagnetic branch decreases as a function of field, the energy of the ferromagnetic branch increases as a function of field.

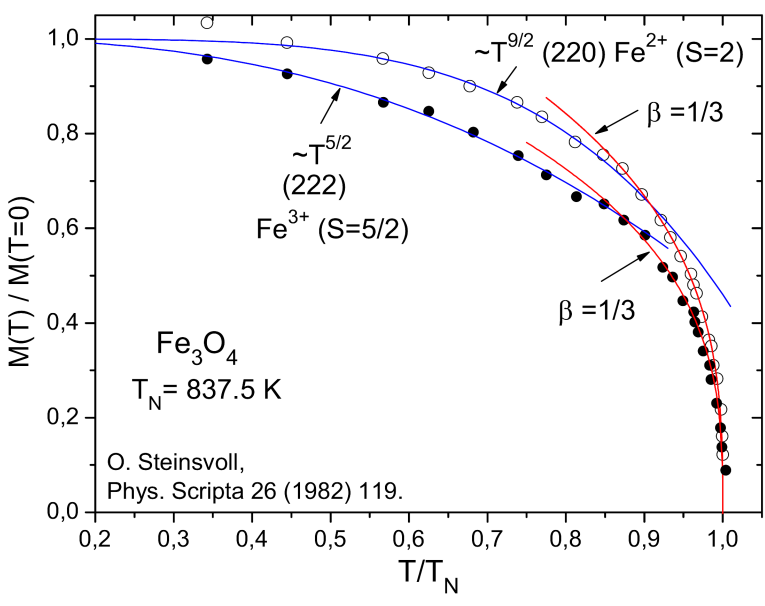

Fig. 3. Order parameters of the $\mathrm{FeO}(S=2$, circles) and of the $\mathrm{Fe}_{2} \mathrm{O}_{3}(S=5 / 2$, dots $)$ subsystem in $\mathrm{Fe}_{3} \mathrm{O}_{4}$ sampled individually by (220) and by (222) magnetic scattering intensity, respectively, as a function of reduced temperature [12]. The different temperature dependencies indicate decoupling of the two subsystems. The boson fields that control thermal decrease of the order parameter have vector character and have different dimensionalities along different crystallographic directions.

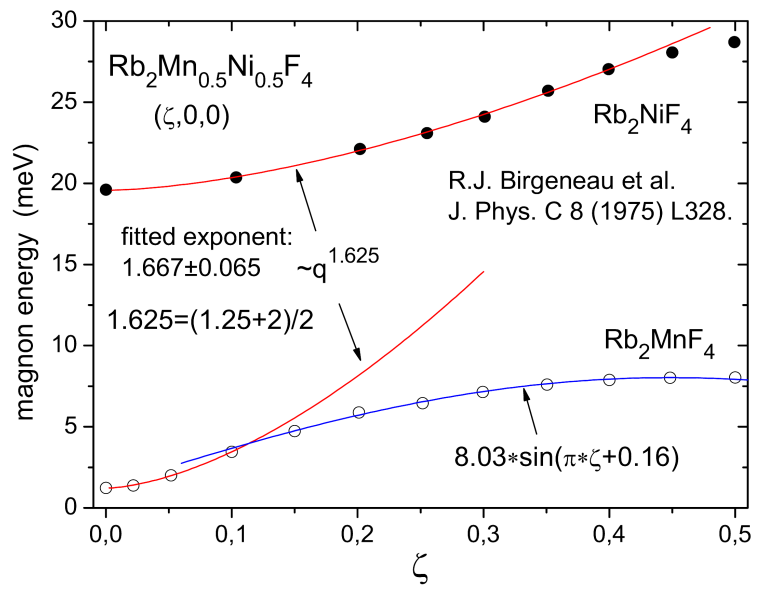

Fig. 4. The two magnon branches (along a-axis) of $\mathrm{Rb}_{2} \mathrm{Mn}_{0.5} \mathrm{Ni}_{0.5} \mathrm{~F}_{4}$ can be attributed to the $\mathrm{Rb}_{2} \mathrm{NiF}_{4}$ $\left(T_{N}=94.5 \mathrm{~K}\right)$ and to the $\mathrm{Rb}_{2} \mathrm{MnF}_{4}\left(T_{N}=38.4 \mathrm{~K}\right)$ subsystem [14]. The pure materials have gap energies of $4.28 \mathrm{meV}\left(\mathrm{Rb}_{2} \mathrm{NiF}_{4}\right)$ and $0.63 \mathrm{meV}\left(\mathrm{Rb}_{2} \mathrm{MnF}_{4}\right)$. The enhanced magnon gap energies are indicative of increased magnon-boson interactions. Magnon dispersion of the $\mathrm{Rb}_{2} \mathrm{MnF}_{4}$ branch displays analytical crossover from power function to sine function of wave vector (see text).

For EuTe [10], for $\mathrm{CoCl}_{2}$ and for $\mathrm{CoBr}_{2}$ [8] measurements of the two magnon gap energies as a function of a magnetic field are available. Figure 2 shows AFMR data of the two gap energies of $\mathrm{CoCl}_{2}$ [8]. In $\mathrm{CoCl}_{2}$ the ferromagnetic planes are stacked antiferromagnetically along hexagonal c-axis [11]. The field dependence of the highenergy gap has much similarity with the critical field of an antiferromagnet and characterizes the upper magnon 


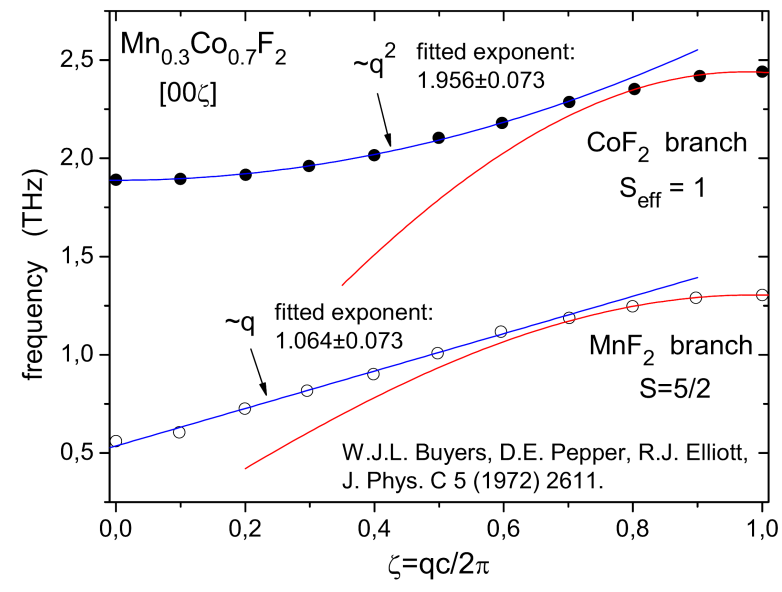

Fig. 5. A qualitatively similar result as for $\mathrm{Rb}_{2} \mathrm{Mn}_{0.5} \mathrm{Ni}_{0.5} \mathrm{~F}_{4} \quad$ (Fig. 4) is obtained for $\mathrm{Mn}_{0.3} \mathrm{Co}_{0.7} \mathrm{~F}_{2}$ [16]. Upper branch can be attributed to $\mathrm{CoF}_{2}$, lower branch to $\mathrm{MnF}_{2}$. However, absolute magnon energies are considerable different compared to the pure materials. In pure $\mathrm{MnF}_{2}$ magnon energies are in the range $0.266-1.64 \mathrm{THz}$ and in pure $\mathrm{CoF}_{2}$ in the range 1.13-1.94 $\mathrm{THz}[16]$.

branch as antiferromagnetic branch. The low energy gap increases unlimited with field and therefore has to be viewed as ferromagnetic branch.

An ideal material to search for an optical magnon is $\mathrm{Fe}_{3} \mathrm{O}_{4}$ (magnetite) [12, 13]. $\mathrm{Fe}_{3} \mathrm{O}_{4}$ can be considered as an ordered mixture of $\mathrm{FeO}(S=2)$ and of $\mathrm{Fe}_{2} \mathrm{O}_{3}$ (hematite) $(S=5 / 2)$. Accordingly, it is possible to investigate the $\mathrm{FeO}$ sublattice and the $\mathrm{Fe}_{2} \mathrm{O}_{3}$ sublattice individually by observation of (220) and (222) magnetic scattering intensities, respectively. As Fig. 3 shows, the $\mathrm{Fe}_{2} \mathrm{O}_{3}$ order parameter follows $\mathrm{T}^{5 / 2}$ dependence. This is the universality class of the $1 \mathrm{D}$ boson field in magnets with half-integer spin $(S=5 / 2)$. The order parameter of the $\mathrm{FeO}$ subsystem follows the $\mathrm{T}^{9 / 2}$ function of the isotropic boson field in magnets with integer spin $(S=2)$. The different power functions of temperature show that the two subsystems are largely decoupled and are controlled by different boson fields. Surprisingly, the boson fields that control spin dynamics of the FeO and of the $\mathrm{Fe}_{2} \mathrm{O}_{3}$ subsystem have different dimensionalities. This illustrates the vector character of the boson fields and their clearly defined dimensionalities. By chance, the critical exponents of the two universality classes are both $\beta=1 / 3[12]$. Interestingly, to the (111) intensity both subsystems contribute. The order parameter evaluated from (111) intensity exhibits perfect $T^{3.5}$ dependence [13]. The interference exponent of 3.5 can be considered as average of 4.5 and 2.5 of the $\mathrm{FeO}$ and of the $\mathrm{Fe}_{2} \mathrm{O}_{3}$ subsystem, respectively.

Another interesting material with different magnetic atoms is the solid solution $\mathrm{Rb}_{2} \mathrm{Mn}_{0.5} \mathrm{Ni}_{0.5} \mathrm{~F}_{4}$ (Fig. 4) [14]. The high-energy magnon branch can be attributed to the $\mathrm{Rb}_{2} \mathrm{NiF}_{4}$ system $(S=1)$ and the low-energy magnon branch to the $\mathrm{Rb}_{2} \mathrm{MnF}_{4}$ system $(S=5 / 2)$. This view conforms to the larger ordering temperature of $\mathrm{Rb}_{2} \mathrm{NiF}_{4}$ $\left(T_{N}=94.5 \mathrm{~K}\right)$ compared to $T_{N}=38.4 \mathrm{~K}$ of $\mathrm{Rb}_{2} \mathrm{MnF}_{4}$ and to the larger gap energy of $\mathrm{Rb}_{2} \mathrm{NiF}_{4}(4.28 \mathrm{meV})$ compared to $0.63 \mathrm{meV}$ for $\mathrm{Rb}_{2} \mathrm{MnF}_{4}$. Note that the highenergy magnon branch always has integer spin. However, in the mixed system the gap energies are much larger than in pure $\mathrm{Rb}_{2} \mathrm{MnF}_{4}$ and in pure $\mathrm{Rb}_{2} \mathrm{NiF}_{4}$. Additionally, for the pure materials the $q^{x}$ sections of the magnon dispersions often are not well resolved [1]. For $\mathrm{Rb}_{2} \mathrm{Mn}_{0.5} \mathrm{Ni}_{0.5} \mathrm{~F}_{4}$ the exponent of $x=1.625$ can be understood as the average of $x=1.25$ for pure $\mathrm{Rb}_{2} \mathrm{NiF}_{4}$ with $S=1$ and of $x=2$ for pure $\mathrm{Rb}_{2} \mathrm{MnF}_{4}$ with $S=5 / 2$, assuming $2 \mathrm{D}$ boson fields for both materials [1]. According to these $\mathrm{x}$-values the order parameters of $\mathrm{Rb}_{2} \mathrm{NiF}_{4}$ and of $\mathrm{Rb}_{2} \mathrm{MnF}_{4}$ should decrease by $\mathrm{T}^{2}$ function and by $\mathrm{T}^{3 / 2}$ function, respectively $[1,4]$. The large gap energies in the mixed system indicate enhanced interactions between magnons and boson field. The interference exponent of $x=1.625$ indicates interactions between the two subsystems.

Data of Fig. 4 show that the $q^{x}$ dispersion holds over a large $q$-range if the gap energy is large. In other words, the gap energy is a measure of the magnon-boson interaction. As the $\mathrm{Rb}_{2} \mathrm{MnF}_{4}$ branch shows, at the limit of the $q^{x}$ function analytical crossover to sine function of wave vector occurs. Interestingly, a phenomenological phase shift has to be applied to the argument of the sine function in order to obtain good agreement with the experimental data. The phase shift seems to be another measure of magnon-boson interaction. A similar observation is made when Debye bosons and acoustic phonons interact [17].

Similar results as for $\mathrm{Rb}_{2} \mathrm{Mn}_{0.5} \mathrm{Ni}_{0.5} \mathrm{~F}_{4}$ are obtained for $\mathrm{Mn}_{0.3} \mathrm{Co}_{0.7} \mathrm{~F}_{2}$ [15] and for $\mathrm{KMn}_{0.29} \mathrm{Co}_{0.71} \mathrm{~F}_{3}$ [16]. Figure 5 shows the high-energy $\mathrm{CoF}_{2}$ magnon branch and the low-energy $\mathrm{MnF}_{2}$ branch of $\mathrm{Mn}_{0.3} \mathrm{Co}_{0.7} \mathrm{~F}_{2}$ [16]. The gap energies of $1.89 \mathrm{THz}$ and $0.56 \mathrm{THz}$ are larger than in the pure materials $(1.13 \mathrm{THz}$ and $0.266 \mathrm{THz}$, respectively). The fitted exponents $x=2$ and $x=1$ indicate isotropic boson fields for integer spin $\left(\mathrm{S}_{\text {eff }}=1\right)[18]$ and for half-integer spin $(S=5 / 2)[1,4]$. Analytical crossover from power function to sine function of wave vector (including phase shift) is evident.

\section{Summary}

In magnetic systems with two in-equivalent magnetic atoms, two magnon branches commonly are observed. The two magnon branches can be attributed to the constituent materials, although they have modified energies. In particular, magnon gap energies in the mixed systems are much larger than in the pure materials [14-16]. The upper magnon branch always is due to the component with integer spin and larger gap energy [1]. The large gap energies indicate that magnon-boson interactions are much stronger than can be anticipated for the exchange interactions between the in-equivalent magnetic moments. Under this condition, optical magnons seem not possible. 


\section{References}

[1] U. Köbler, Acta Phys. Pol. A 127, 1694 (2015).

[2] K.G. Wilson, J. Kogut, Phys. Rep. 12C, 75 (1974).

[3] A. Hoser, U. Köbler, Acta Phys. Pol. A 127, 350 (2015).

[4] A. Hoser, U. Köbler, J. Physics: Conf. Series $\mathbf{7 4 6}$ 012062 (2016).

[5] B.V. Karpenko, A.V. Kuznetsov, V.V. Dyakin, J. Magn. Magn. Mater. 117, 317 (1992).

[6] M.P.H. Thurlings, E. Frikkee, H.W. de Wijn, Phys. Rev. B 25, 4750 (1982).

[7] G. Pepy, J. Phys. Chem. Solids 35, 433 (1974).

[8] J. Magarińo, J. Tuchendler, A.R. Fert, J. Gelard, Sol. State Commun. 23, 175 (1977).

[9] E.J. Samuelsen, G. Shirane, Phys. Status Solidi 42, $241(1970)$.
[10] S.O. Demokritov, N.M. Kreines, V.I. Kudinov, Sov. Phys. JETP 65, 389 (1987).

[11] M.T. Hutchings, J. Phys. C 6, 3143 (1973).

[12] O. Steinsvoll, Physica Scripta 26, 119 (1982).

[13] A.W. McReynolds, T. Riste, Phys. Rev. 95, 1161 (1954).

[14] R.J. Birgeneau, L.R. Walker, H.J. Guggenheim, J. Als-Nielsen, G. Shirane, J. Phys. C 8, L328 (1975).

[15] W.J.L. Buyers, T.M. Holden, E.C. Svensson, R.A. Cowley, R.W.H. Stevenson, Phys. Rev. Lett. 27, 1442 (1971).

[16] W.J.L. Buyers, D.E. Pepper, R.J. Elliott, J. Phys. C 5, 2611 (1972).

[17] U. Köbler, Int. J. Thermodyn. 18, 277 (2015).

[18] U. Köbler, A. Hoser, J.-U. Hoffmann, Physica B 382, 98 (2006). 